\title{
E layer dominated ionosphere observed by EISCAT/ESR radars during solar minimum
}

\author{
Hongtao Cai ${ }^{1,2}$, Fei Li ${ }^{1,2}$, Ge Shen ${ }^{1,2}$, Weijia Zhan ${ }^{1,2}$, Kangjun Zhou ${ }^{1,2}$, Ian Willian McCrea ${ }^{3}$, and Shuying Ma ${ }^{1,2}$ \\ ${ }^{1}$ Department of Space Physics, School of Electronic Information, Wuhan University, Wuhan, 430072, China \\ ${ }^{2}$ Key Laboratory of Geospace Environment and Geodesy, Ministry of Education of PRC, Wuhan, 430072, China \\ ${ }^{3}$ Space Science and Technique Department, Rutherford Appleton Laboratory, Chilton, Oxfordshire, OX11 0QX, UK
}

Correspondence to: Hongtao Cai (htcai@whu.edu.cn)

Received: 3 January 2014 - Revised: 22 July 2014 - Accepted: 8 September 2014 - Published: 10 October 2014

\begin{abstract}
According to the study by Mayer and Jakowski (2009), periods of E layer dominated ionosphere (ELDI) are defined as being characterized by vertical electron density profiles having a maximum density at E layer altitudes. In this paper, characteristics of ELDI intervals have been investigated, focusing on their temporal variations, using field-aligned measurements from the EISCAT and ESR radars during the interval 2009-2011. ELDI events were identified using simple but reasonable criteria, in which a minimal duration was required to exclude possible "fake" events induced by random errors in measurements. It was found that ELDIs were observed more often in winter and earlier spring than other seasons, especially in the auroral zone. The occurrence of ELDI intervals peaks around geomagnetic midnight at auroral latitudes, while it reaches a maximum around geomagnetic local noon at the latitude of the ESR. Our results imply that ELDI intervals appear to be a sporadic rather than a regular phenomenon, in disagreement with previous results inferred from radio occultation measurements. The discrepancy between the typical durations of ELDI events observed by the two radars is remarkable, being $30 \mathrm{~min}$ on average at Troms $\varnothing$ but about a half of this at Svalbard. During intervals of ELDI, the mean thicknesses of the $\mathrm{E}$ layer are quite close at the two sites, as are the values of $\mathrm{H}_{\mathrm{m}} \mathrm{E}$ and the ratio of $\mathrm{N}_{\mathrm{m}} \mathrm{E} / \mathrm{N}_{\mathrm{m}} \mathrm{F}_{2}$. Case studies confirm that either extra $\mathrm{E}$ layer ionization or F layer density depletion alone could lead to the presence of ELDIs. Based on a careful check on ELDI intervals of various types, however, we suggest that both of them play a critical role in ELDI formation.
\end{abstract}

Keywords. Ionosphere (ionospheric irregularities; polar ionosphere)

\section{Introduction}

The terrestrial ionosphere is formed by the ionization of the atmosphere, due to solar EUV and X-ray radiations and, mainly at high-latitudes, by energetic particles impinging on the atmosphere from the magnetosphere. The ionosphere is coupled closely with both the magnetosphere and the thermosphere in which it is immersed. This leads the ionosphere to vary significantly and a number of anomalies have been reported. Some of these anomalies arise mainly due to temporal variations, such as the winter anomaly and the semiannual anomaly (e.g. Yonezawa and Arima, 1959; Yonezawa, 1971; Cai et al., 2007). There are also some phenomena related to ionospheric spatial structure, including the equatorial anomaly (e.g. Liang, 1947), sporadic E ( $\left.E_{s}\right)$ (e.g. Mathews, 1998) and spread F (e.g. Farley et al., 1970). Recently, Mayer and Jakowski (2009) found that ionospheric profiles in the polar region sometimes have their maximum density at E layer altitude, which was identified as another class of anomaly: E layer dominant ionosphere (ELDI). $\mathrm{E}_{\mathrm{s}}$ formation or other types of ionization were not distinguished in the concept of ELDI. Investigations of these ionospheric anomalies are not only of basic importance in understanding and forecasting space weather, but also have significant applications in radio propagation and communication.

To our knowledge, work on ELDIs has been reported comparatively little in the literature. Their global distribution in the Northern Hemisphere as well as their spatial distribution and solar-cycle variability were investigated by Mayer and Jakowski (2009) using electron density profiles retrieved from GPS radio occultation (RO) measurements from the COSMIC and CHAMP satellites. It was found that ELDI 
occurrence was distributed symmetrically around the magnetic pole and that the shape of the spatial distribution could be approximated by an ellipse. The contribution of geomagnetic activity to ELDI occurrence was found to be significant, especially during nighttime.

Four years later, Wu et al. (2013) extended the work by comparing the spatial distributions of ELDIs in the Arctic and Antarctic during solar minimum. Four-year RO measurements from COSMIC in winter during the period 20072010 were used. It was found that the spatial distribution of ELDI coincided with the location of the auroral oval for both hemispheres. The authors claimed that these observed ELDIs were mainly caused by energetic particle precipitation in the auroral zone. Based on RO measurements, Wu et al. (2013) found that ELDI occurrence was up to $90 \%$ in the nighttime auroral zone, whilst Mayer and Jakowski (2009) claimed that ELDI was a regular phenomenon in darkness.

Previous reports about ELDIs have mainly focused on their spatial distribution, whilst their temporal variations are poorly understood. Because of the way that satellite data are sampled, temporal resolution at a fixed point is rather low and it is hard to gain a clear picture of the process from the appearance to the disappearance of an ELDI from RO measurements alone. In addition, the seasonal and diurnal variations of ELDIs are still not clear, though these are important for understanding the formation mechanism of ELDIs.

Ground-based incoherent scatter (IS) radar can provide successive electron density vertical profiles with rather high time resolution. The European Incoherent Scatter (EISCAT; $69.6^{\circ} \mathrm{N}, 19.2^{\circ} \mathrm{E}$ ) radar is located beneath the auroral oval for an extended interval, whilst the EISCAT Svalbard radar (ESR; $78^{\circ} \mathrm{N}, 16^{\circ} \mathrm{E}$ ) crosses the cusp during the daytime and lies within the polar cap during the nighttime (e.g. Cai et al., 2007). Both of the radars are designed to regularly probe ionospheric properties, including electron density and temperature, along the local geomagnetic field line, with about 2 -minute resolution. The operating modes of the two radars thus make it possible for us to investigate the characteristics of ELDIs from a new viewpoint, focusing on their seasonal variation, diurnal variation and duration.

In this paper, a survey of ELDI characteristics is carried out with help of field-aligned measurements from the EISCAT UHF radar at Troms $\varnothing$ and the ESR $42 \mathrm{~m}$ dish at Svalbard. Our work reveals some novel features and sheds more light on the possible formation mechanism of ELDIs. The data set used in the present work and the criteria used to define ELDI are introduced in Sect. 2. Detailed results are presented in Sect. 3 and discussed in Sect. 4, followed by a brief summary.

\section{Data analysis}

Field-aligned measurements made by the EISCAT/ESR radars during 2009-2011 are used in the present work.
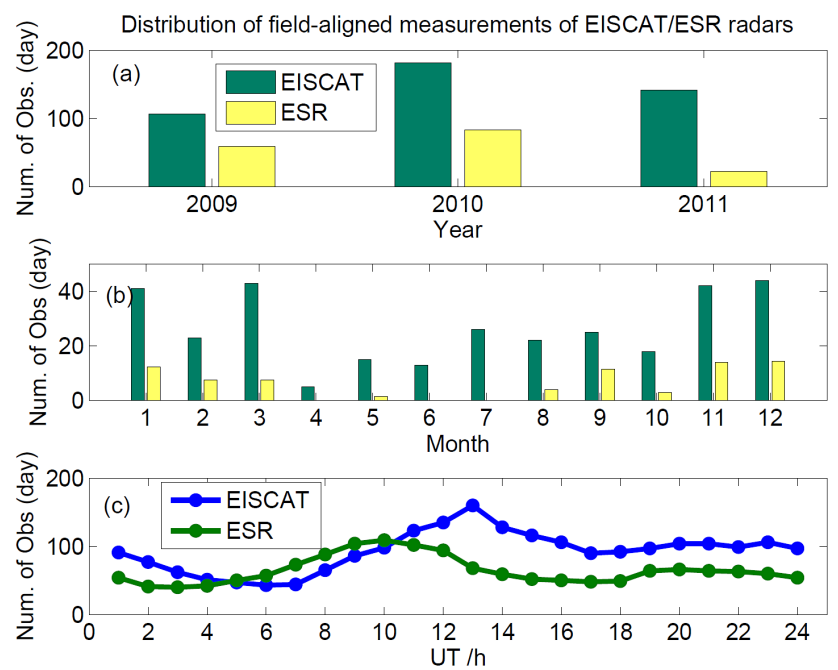

Figure 1. Distribution of field-aligned measurements from EISCAT/ESR radars with (a) year, (b) month and (c) UT $(\mathrm{LT} \approx \mathrm{UT}+1 \mathrm{~h}, \mathrm{MLT} \approx \mathrm{UT}+3 \mathrm{~h})$.

Figure 1 displays numbers of EISCAT/ESR observation periods, which have been used in present study, broken down by year (panel a), month (panel b) and UT (panel c), respectively. Only time sequences of successive radar measurements with lengths between $4 \mathrm{~h}$ and 1 day are included. There are far fewer field-aligned observations available for April, June and July from the ESR site. For Troms $\varnothing$, however, there are more than 20 days of field-aligned measurements in most months, with only April having fewer than 10 days (Fig. 1b). This data set provides us a good opportunity to figure out the seasonal variation of ELDI occurrence. Most of the observations were made under geomagnetic quiet conditions and only 3 days of measurements from the EISCAT radar were accomplished during days with a Dst index lower than $-101 \mathrm{nT}$.

Figure 1c shows the number of observation intervals as a function of UT for both radars. It is easy to calculate the approximate magnetic local time from MLT $\approx \mathrm{UT}+3 \mathrm{~h}$ and the local time from $\mathrm{LT} \approx \mathrm{UT}+1 \mathrm{~h}$. The diurnal coverage of radar measurements, while somewhat variable for both radars, is sufficient even for a study of this kind. For most universal times, there are more than 50 days of radar measurements. For the EISCAT radar, the number of observations minimizes at 07:00 UT (10:00 MLT) and has a peak of 160 days at 13:00 UT (16:00 MLT). At the ESR site, the peak number of radar observations appears slightly earlier, at around 10:00 UT (13:00 MLT) and the minimum is around 03:00 UT (06:00 MLT).

The signal-to-noise ratio (SNR) indicates the reliability of the data and is therefore important in our analysis. Unreliable results have been eliminated, based on the fitting flags of the radar measurements. In addition, outliers of larger than 
$3 \sigma$ were also removed in order to avoid possible mistakes in identifying the density peaks of the $\mathrm{E}$ layer and $\mathrm{F}$ layer.

In present work, an ELDI event was identified from successive time series of electron density profiles, provided that all of the following conditions were met:

1. Peak density of the $\mathrm{E}$ layer $\left(\mathrm{N}_{\mathrm{m}} \mathrm{E}\right)$ is larger than that of the $\mathrm{F}$ layer $\left(\mathrm{N}_{\mathrm{m}} \mathrm{F}_{2}\right)$.

2. Peak height of the $E$ layer $\left(\mathrm{H}_{\mathrm{m}} \mathrm{E}\right)$ is in the range of 90 $140 \mathrm{~km}$.

3. Duration of the event is no shorter than $6 \mathrm{~min}$.

The first two criteria are basically similar to those adopted by Mayer and Jakowski (2009) and Wu et al. (2013), whilst the last one has been applied to exclude possible "fake" events induced by random errors in observations. Compared to radio occultation measurements, the advantage of ground-based instrument observations is their capability to determine the duration of an ELDI event. The time resolution of the radar measurements is $2 \mathrm{~min}$. The minimal ELDI duration of $6 \mathrm{~min}$ was adopted somewhat arbitrarily, although it did a good job in practice, as we will show in the following sections.

Once an ELDI event was identified, some important properties were measured and recorded for further analysis. Figure 2 shows an illustration of an ELDI profile. The peak densities $\left(\mathrm{N}_{\mathrm{m}} \mathrm{F}_{2}\right.$ and $\left.\mathrm{N}_{\mathrm{m}} \mathrm{E}\right)$ and peak heights $\left(\mathrm{H}_{\mathrm{m}} \mathrm{F}_{2}\right.$ and $\left.\mathrm{H}_{\mathrm{m}} \mathrm{E}\right)$ are defined as usual. The thickness of the $\mathrm{E}$ layer is defined as being the difference between the two altitudes at which the electron density firstly drops to 70 percent of $\mathrm{N}_{\mathrm{m}} \mathrm{E}$, upwards and downwards. In addition, we define the ratio of $\mathrm{N}_{\mathrm{m}} \mathrm{E}$ and $\mathrm{N}_{\mathrm{m}} \mathrm{F}_{2}$ during an ELDI event:

$r_{\mathrm{EF}}=\frac{\mathrm{N}_{\mathrm{m}} \mathrm{E}}{\mathrm{N}_{\mathrm{m}} \mathrm{F}_{2}}$.

According to the definition of ELDI, the value of $r_{\mathrm{EF}}$ should be larger than one. To some extent, the parameter $r_{\mathrm{EF}}$ quantifies the magnitude of an ELDI.

\section{Observations}

We identified 428 and 164 valid ELDI events from the fieldaligned measurements during 2009-2011 of the EISCAT UHF and ESR radars, respectively. Effects from geomagnetic activity are negligible, since this period covers a prolonged solar minimum, and only 8 ELDI events were detected by EISCAT radar during 3 days with a Dst index lower than $-101 \mathrm{nT}$. Individual checks of these storm-time ELDIs revealed no remarkable difference from the others. The number of ELDI intervals over Svalbard is far less than that at Troms $\varnothing$, partly owing to the smaller number of observations from the ESR site (Fig. 1a).

In the following subsections, a survey of these ELDI events and their properties is presented.

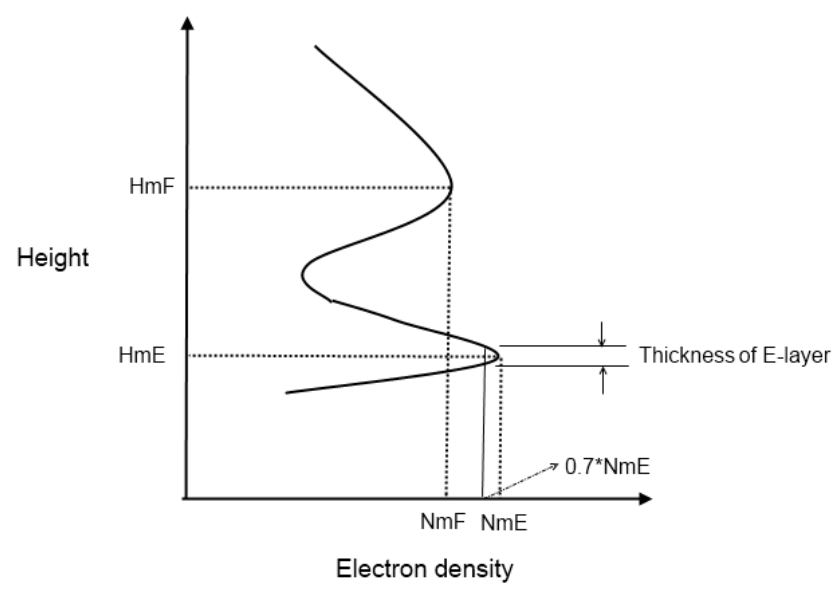

Figure 2. An illustration of some characteristics parameters during the presence of ELDI. See text for details.

\subsection{Occurrence of ELDI}

The number of ELDIs in each month observed by EISCAT/ESR radars is displayed in Fig. 3a. Generally, more ELDI events were recorded by the EISCAT mainland radar. Readers must keep in mind that the numbers of field-aligned measurements available in each month are somewhat variable between the two radars (Fig. 1b).

The relative number of ELDIs per day in each month is depicted in Fig. 3b. This has been deduced by normalizing the number of ELDIs (Fig. 3a) by the available radar observations (Fig. 1b). To some extent, it could be regarded as a good indicator of the seasonal variation of ELDI occurrence in the auroral zone, bearing in mind that the field-aligned measurements of EISCAT radar have a fairly good coverage during most months (Fig. 1b).

From Fig. 3b, it is clear that there is a relatively higher ELDI occurrence in winter and earlier spring, namely in November, December, January, February and March, for both radars. It seems, however, that ELDIs have a much lower occurrence rate in the remaining months. There appears to be an exception in May for ESR observations, but careful checking suggests that this may be an unreliable result due to the low number of ESR observations available during that month (Fig. 1b). Indeed, the apparent seasonal variation of ELDI at ESR site should be interpreted with caution, because of quite poor observational coverage from April to August (Fig. 1b).

Because both radars have relatively better coverage in winter and earlier spring (Fig. 1b), the following subsections focus only on ELDIs observed during those months, namely from November to March. The remaining data set comprises 389 events from the EISCAT mainland site and 144 at ESR. The effect on the overall database is very slight, since over $90 \%(88 \%)$ of all ELDI events were recorded by the EISCAT mainland (ESR) radar during these months. 

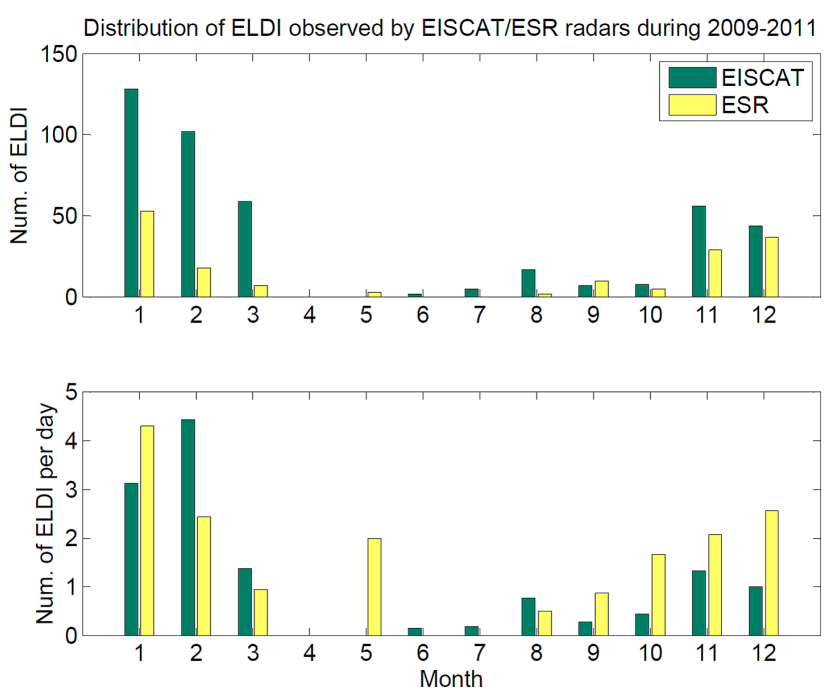

Figure 3. Distribution of ELDI observed by EISCAT/ESR radars during 2009-2011. The top panel denotes for absolute number of ELDI detected by radars and the bottom panel for normalized number of ELDIs with observations in Fig. $1 b$.

\subsection{Duration of ELDI}

The top panel of Fig. 4 shows the duration of each ELDI event observed by the EISCAT UHF radar at Troms $\varnothing$ during the period from November to March. It can be seen that the typical duration of these events varies from a couple of minutes to hours. The variation in duration is very similar for the ESR site (not shown here).

The distribution of ELDI duration is displayed in the bottom panel of Fig. 4 for both radars, using 2-minute bins. The bin size of 2 minutes was chosen to match the time resolution of the radar measurements. About one-third of the ELDI events observed by both radars last for an interval just above the lower threshold of 6 minutes. The other two-thirds last for a longer period, though most of them fall into bins no longer than $30 \mathrm{~min}$. A number of the ELDI intervals detected by the EISCAT mainland radar have durations longer than an hour. Detailed checks indicate that most of these long-lasting ELDIs were observed in December, January and February around geomagnetic midnight (18:0002:00 UT, 21:00-05:00 MLT).

Overall, the average duration of ELDIs is $30 \mathrm{~min}$ at Troms $\varnothing$ and $14 \mathrm{~min}$ at Svalbard, respectively, which is more than twice the threshold introduced in our criteria for identifying an ELDI. This suggests that our definition of this threshold is reasonable in practice.

\subsection{E layer thickness of and $H_{m} E$ during ELDI}

During the presence of ELDI, E layer thickness and $\mathrm{H}_{\mathrm{m}} \mathrm{E}$ were evaluated for each of the measured profiles and then averaged for the event. The distribution of $E$ layer thicknesses
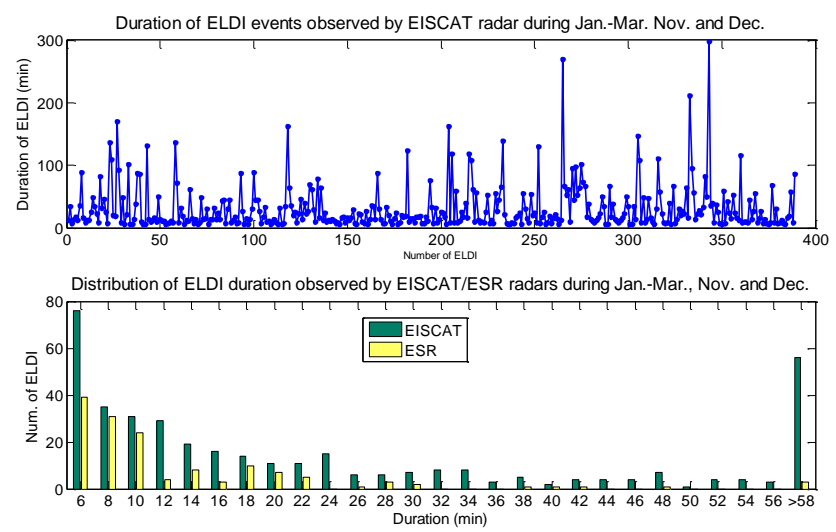

Figure 4. Variation of ELDI duration inferred from EISCAT radar measurements (the upper panel) and distribution from both radars (the bottom panel).

during ELDI events is displayed in the top panel of Fig. 5. The bin size in these distributions is $3 \mathrm{~km}$, which matches the altitude resolution of the radar measurements in the $\mathrm{E}$ region. Solid lines, showing a fitted Gaussian distribution, are superposed for reference, with residuals in form of error bars. According to the fitting results, the $\mathrm{E}$ layer thickness has a peak value of $21 \mathrm{~km}$ at the EISCAT mainland radar, compared to $17.8 \mathrm{~km}$ at the ESR. The values of parameter $c$ in the top panel indicate that the E layer thickness detected by the ESR almost matches that for the EISCAT mainland observations, within the one sigma confidence interval. Considering the altitude resolution of $3 \mathrm{~km}$ in radar measurements, it seems that the mean values of $E$ layer thickness during ELDI events are effectively indistinguishable between the two sites.

The bottom panel of Fig. 5 shows the distribution of $\mathrm{H}_{\mathrm{m}} \mathrm{E}$ during ELDI events. Once again, the step size is $3 \mathrm{~km}$ and lines of Gaussian fitting are displayed for reference. The predominant value of $\mathrm{H}_{\mathrm{m}} \mathrm{E}$ inferred from EISCAT mainland measurements is quite clear, being $114.7 \mathrm{~km}$, with standard deviation of $11.2 \mathrm{~km}$. The peak heights of the ELDIs observed by ESR radar, however, are widely scattered throughout the plotted range, with a weak peak at around $110.4 \mathrm{~km}$. The difference in the two distributions may be partly explained by the comparatively smaller number of ESR observations, resulting in many fewer ELDI events being available for analysis.

\section{$3.4 \quad r_{\mathrm{EF}}$ during ELDI}

Figure 6 shows the distribution of $r_{\mathrm{EF}}$ for the observed ELDI events. The step of these bins is 0.1 . There are reasonably well-defined maxima for both radars, with the number of ELDIs reducing gradually on both sides. Gaussian fitting has once again been carried out for measurements from the two radars, as superposed in the figure. Residuals are presented as error bars. 

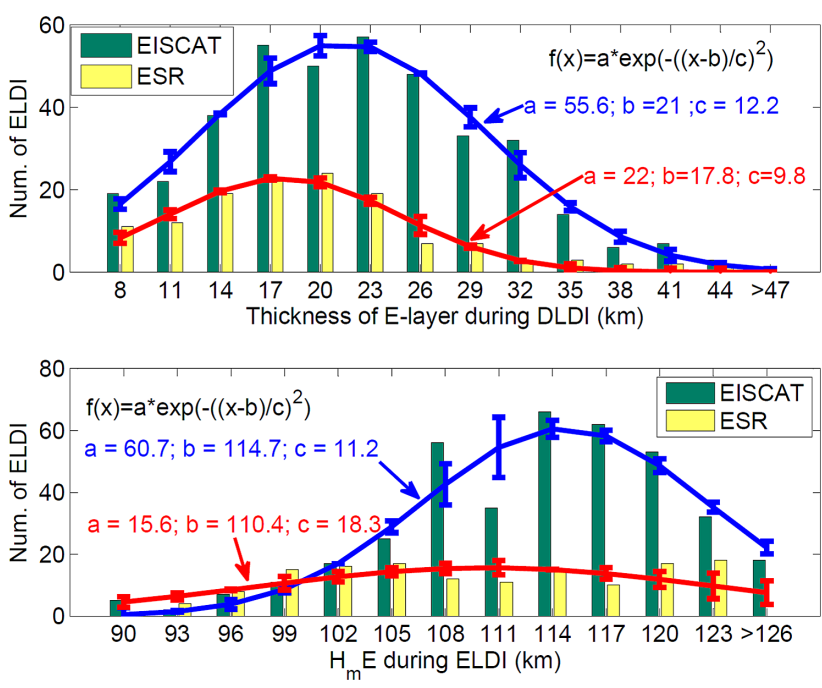

Figure 5. Distribution of ELDI with E layer thickness (the top panel) and $\mathrm{H}_{\mathrm{m}} \mathrm{E}$ (the bottom panel). Gaussian fitting lines of the distributions are also superposed, with residuals as error bars.

The fitting curves exhibit a predominant peak at $r_{\mathrm{EF}}=1.5$ for the EISCAT mainland radar, compared to 1.3 for the ESR. The standard deviations of the fitted curves are 0.6 and 0.4 , respectively, suggesting that the dominant value for $r_{\mathrm{EF}}$ is basically similar for the two sites, within the one sigma confidence interval.

The values of parameter $b$ in the figure would also tend to suggest that the abnormal vertical structure of density profiles during the presence of ELDIs is quite clear, as will be shown later in Fig. 9, with $\mathrm{N}_{\mathrm{m}}$ E being up to $50 \%$ higher than $\mathrm{N}_{\mathrm{m}} \mathrm{F}_{2}$.

\section{Discussions}

\subsection{Diurnal variation of ELDI occurrence}

Based on 4 years of radio occultation measurements from COSMIC, Wu et al. (2013) found that the occurrence of ELDI was higher on the nightside and was rare during the period 12:00-15:00 MLT in the dayside sector. The diurnal variation of ELDI occurrence observed by the EISCAT/ESR radars is depicted in the top panel of Fig. 7, whilst the normalized occurrence of ELDI as a function of time is also shown in the bottom panel. This was achieved by normalizing number of ELDIs to the number of observations in every hour (Fig. 1c). The average number displayed in the bottom panel, therefore, can be understood as the diurnal variation of ELDI occurrence. The figure is plotted as a function of universal time (UT) for both radars. It is easy to get approximate magnetic local time, using the formula MLT $\approx \mathrm{UT}+3 \mathrm{~h}$ and local time with the formula $\mathrm{LT} \approx \mathrm{UT}+1 \mathrm{~h}$.

It is clear that the peak occurrence times of ELDIs observed by the EISCAT mainland and ESR radars are quite

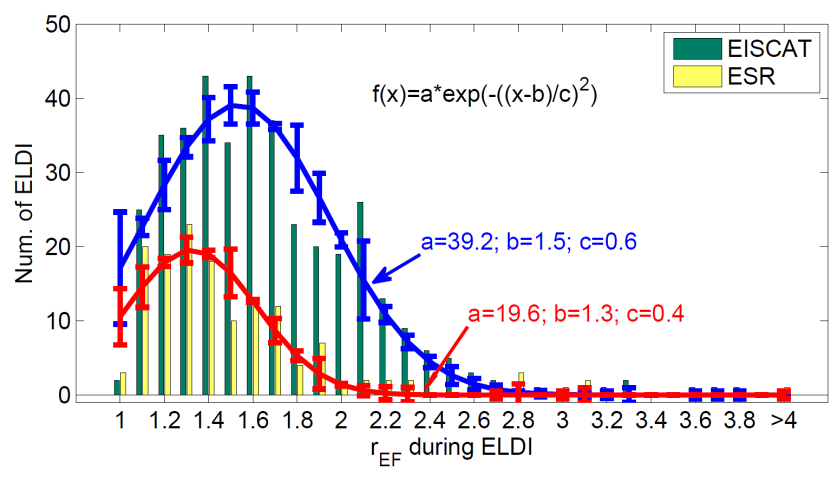

Figure 6. Distribution of ELDI counts with $r_{\mathrm{EF}}$ observed by EISCAT/ESR radars. Gaussian fitting lines were superposed for reference.

different. In the auroral zone (i.e. at the EISCAT mainland site), ELDI intervals are most frequently observed during the interval from 20:00 to 02:00 UT (23:00 to 05:00 MLT). This agrees with the results reported by Wu et al. (2013). For ESR measurements, however, the maximum is around MLT noon (08:00 UT, 11:00 MLT), with a hint of a secondary peak in the pre-midnight sector (19:00 UT, 22:00 MLT). This result disagrees with the results inferred from radio occultation measurements (Wu et al., 2013).

An understanding of particle precipitation in the polar region helps us to make sense of the results. Under geomagnetic quiet conditions, the ESR radar is in the cusp region around noon and in the polar cap on the nightside, whilst the EISCAT mainland radar is within the auroral zone during most times of the day. During the interval in which the radar site is located under the auroral oval, precipitating particles of magnetospheric origin would cause additional ionization at $E$ layer altitudes, thus favouring the formation of an ELDI.

It is also interesting to mention that there is a gap of ELDI occurrence in 08:00-15:00 UT (11:00-18:00 MLT) for the EISCAT mainland radars, during which period the maximum of radar observations appears (Fig. 1c).

Our results suggest that the characteristic diurnal variations of ELDI occurrence exhibit remarkable spatial variations, implying that ELDIs are likely to be local rather than global in nature.

\subsection{Is ELDI a regular phenomenon in a dark polar region?}

Based on their radio occultation measurements, Mayer and Jakowski (2009) claimed that ELDI is a regular phenomenon in the nighttime auroral zone, since ELDI signatures were found in up to $80 \%$ of all measured profiles between 07:00 and 08:00 local time during geomagnetic quiet conditions. Wu et al. (2013) came to a similar conclusion, showing that the occurrence rates of ELDI were up to 70 and $90 \%$ for the Arctic and Antarctic, respectively. 

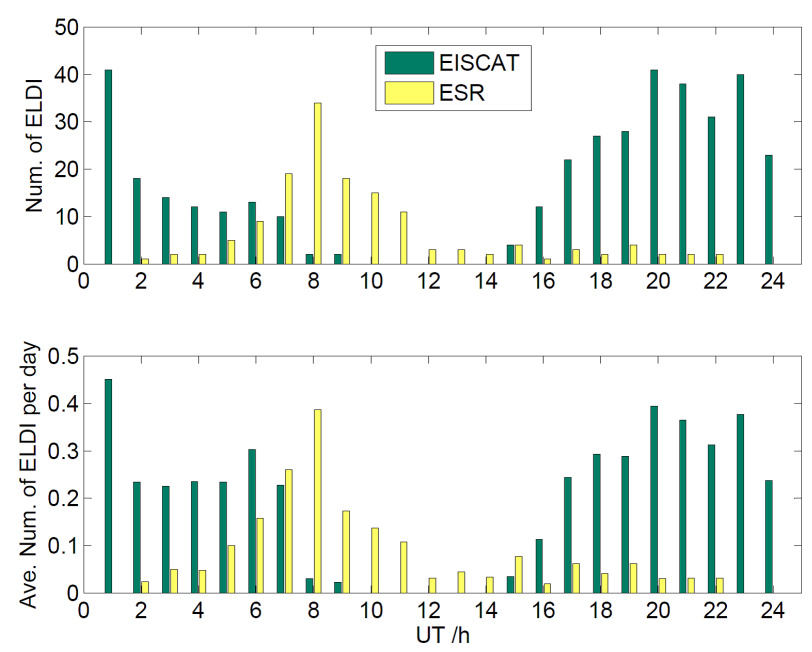

Figure 7. Diurnal variation of ELDIs observed by EISCAT/ESR radars (the top panel). In the bottom panel, numbers of ELDI were normalized according to observation as in Fig. $1 \mathrm{c}(\mathrm{LT} \approx \mathrm{UT}+1 \mathrm{~h}$, $\mathrm{MLT} \approx \mathrm{UT}+3 \mathrm{~h})$.

Ground-based IS radars measurements showed, however, that typically around three ELDIs were recorded during each $24 \mathrm{~h}$ in the auroral zone (the bottom panel of Fig. 3). In February, up to four events were caught per day on average. As far as diurnal variation is concerned (the bottom panel of Fig. 7), the maximum occurrence of ELDIs is around $40 \%$ for both sites. During most local times on the nightside, ELDI occurrence is not higher than $30 \%$ for the EISCAT mainland radar. It is much lower at ESR, being only $5 \%$ in the polar cap. Additionally, ELDIs are rare in the auroral zone around geomagnetic local noon (08:00-14:00 UT, 11:0017:00 MLT), during which period the availability of fieldaligned observations maximizes (Fig. 1c). Ground-based IS radar measurements, therefore, strongly suggest that ELDIs appear sporadically, rather than regularly, in the polar region.

Based on this data set, the contribution of geomagnetic activity to ELDI occurrence appears negligible. Only 8 of the 428 ELDIs were detected by EISCAT radar during storms, namely 5 ELDIs in March, 2 in August and 1 in November. Six of the storm-time ELDIs were seen during the interval 15:00-17:00 UT (18:00-20:00 MLT) and the remaining two were detected at dawn (00:00-02:00 UT, 03:00-05:00 MLT). None of these storm-time ELDIs showed any significantly different characteristics from the others.

It is worth reminding the reader that a minimal duration of ELDI was adopted in our work, so that profiles with transient signature of ELDI were excluded from our results. The duration of ELDI events was not considered in previous reports (Mayer and Jakowski, 2009; Wu et al., 2013) since temporal resolution in satellite measurements is rather poor for any fixed point. The requirement for a minimum event duration would, to some extent, lower the number of ELDIs identified from radar measurements and thus may also change the occurrence statistics of ELDIs.

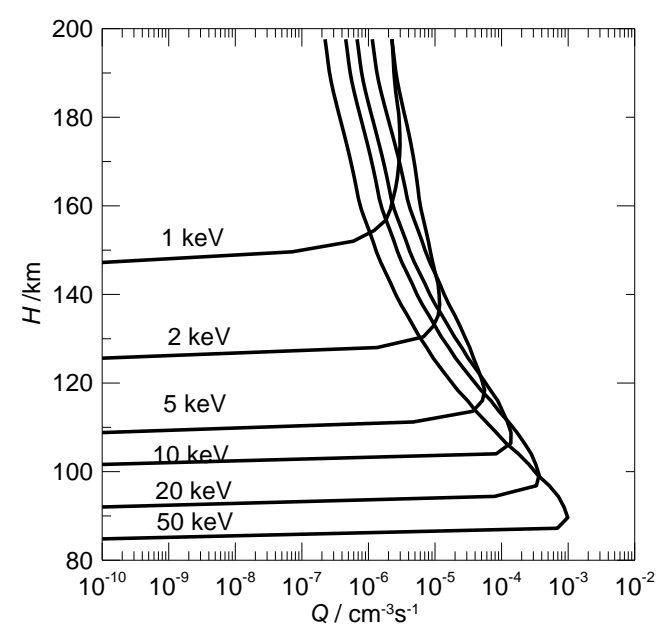

Figure 8. Ionization rate caused by incident monoenergy auroral electrons in polar upper atmosphere (from Cai and Ma, 2007).

\subsection{Possible causes for presence of ELDI}

The electron continuity equation can be written as

$\frac{\partial \mathrm{N}_{\mathrm{e}}}{\partial t}=q_{\mathrm{solar}}+q_{\mathrm{hp}}-\operatorname{div}\left(\mathrm{N}_{\mathrm{e}} \cdot v\right)-L_{\mathrm{e}}$,

in which $q_{\text {solar }}$ represents ionization caused by solar UV/EUV radiations and $q_{\mathrm{hp}}$ the additional ionization from particle precipitation. The third term on the right-hand side corresponds to electron density variations induced by transport, whilst the last item represents the loss due to chemical processes. During the winter, the EISCAT mainland and ESR radars are in darkness during most of the time. Thus the contribution from solar radiation can be reasonably neglected. In the polar region, transport processes, including ambipolar diffusion, wind-induced drifts along the field lines and electrodynamic drifts cross the magnetic field, are important in the $\mathrm{F}$ layer, but could be ignored in the $\mathrm{E}$ layer (Cai and $\mathrm{Ma}$, 2007).

As is well known, precipitating electrons with energy higher than $1 \mathrm{keV}$ will cause their maximum ionization at altitudes below $150 \mathrm{~km}$ (e.g. Rees, 1963; Cai and Ma, 2007). This is the main reason why Wu et al. (2013) concluded that it is particle precipitation that causes the presence of ELDI in the dark polar region. Ionization rate profiles caused by incident precipitating electrons in the polar upper atmosphere are depicted in Fig. 8, which is adopted from Cai and Ma (2007). The thicknesses of the ionization peaks caused by monoenergetic precipitating electrons can be roughly estimated from Fig. 8, using our definition of $E$ layer thickness as given in Sect. 2. It varies from about 10 to $20 \mathrm{~km}$, depending on the energy of the auroral electrons, and is comparable with the average E layer thickness of ELDIs which we observe (Fig. 5). Generally, the energy spectra of auroral incident electrons in polar region can be well described by 


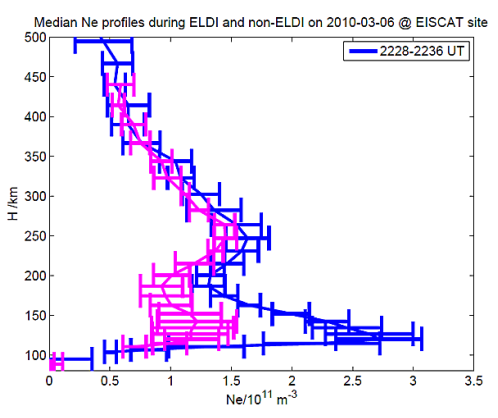

(a)

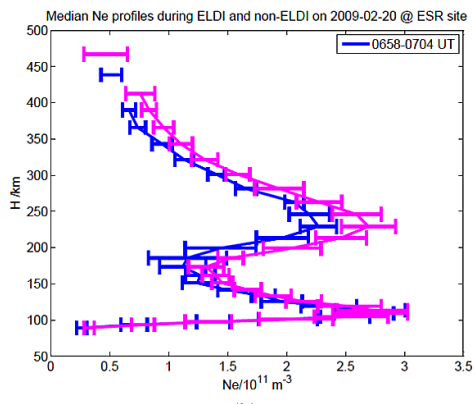

(b)

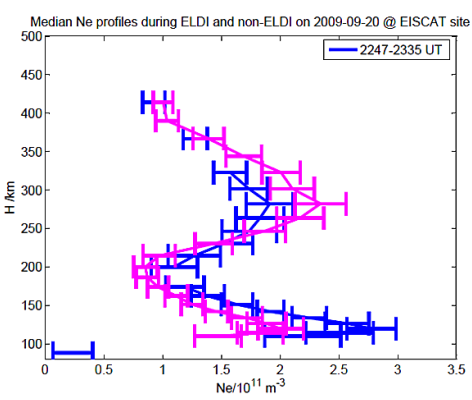

(c)

Figure 9. Electron density median profiles (blue lines) observed by EISCAT/ESR radars during the presence of three types of ELDIs. Ne profiles around presence of the ELDI (magenta lines) are superposed for reference. The standard deviations of these profiles are presented as error bars.

Maxwellian distributions (e.g. Strickland et al., 1989). According to the theory of Rees (1963), incident electrons with a flux peak around $5 \mathrm{keV}$ would induce extra ionization in the E layer at around $110 \mathrm{~km}$ altitude, which coincides with the mean values of ELDI $\mathrm{H}_{\mathrm{m}} \mathrm{E}$, shown in Fig. 5. This type of electron precipitation is frequently observed in the auroral zone (e.g. Burch et al., 1976), and is widely accepted to be accelerated by a field-aligned electric field (e.g. Hultqvist et al., 1999). Provided that the aforementioned auroral precipitation is intense enough, the presence of ELDI would be expected.

ELDIs caused by particle precipitation were indeed observed by the EISCAT radars, and are called type 1 ELDI in this paper. An example, detected in the nighttime auroral region, is displayed in Fig. 9a. The blue line represents the median electron density profile measured during the ELDI and the magenta line indicates the background profile just before or after the ELDI. The background density is determined by the median of a series of profiles during a period with the same time span as the ELDI duration. Standard deviations are superposed as error bars. An enhancement of over $100 \%$ in $\mathrm{N}_{\mathrm{m}} \mathrm{E}$ was detected, while the $\mathrm{N}_{\mathrm{m}} \mathrm{F}_{2}$ remained almost constant during the presence of the ELDI. It is clear that ELDIs of this type are formed mainly due to the abrupt enhancement of $\mathrm{N}_{\mathrm{m}} \mathrm{E}$, in agreement with the hypothesis proposed by $\mathrm{Wu}$ et al. (2013).

For a typical electron density profile in the polar region, $\mathrm{N}_{\mathrm{m}} \mathrm{F}_{2}$ dominates the ionosphere and is usually far greater than $\mathrm{N}_{\mathrm{m}} \mathrm{E}$ (e.g. Cai et al., 2007). For this initial condition, ELDI formation requires either:

1. reduction in $\mathrm{N}_{\mathrm{m}} \mathrm{F}_{2}$

2. enhancement in $\mathrm{N}_{\mathrm{m}} \mathrm{E}$ or

3. combination of (1) and (2).

In theory, therefore, enhancement of $\mathrm{N}_{\mathrm{m}} \mathrm{E}$ might not always be the predominant mechanism for ELDI formation.

The EISCAT mainland and ESR radars also detected ELDIs with different features, as shown in panels $b$ and $c$ of
Table 1. Distributions of three types of ELDIs during 2009-2011.

\begin{tabular}{lrrr}
\hline & Type 1 & Type 2 & Type 3 \\
\hline EISCAT site & $37 \%$ & $28 \%$ & $35 \%$ \\
ESR site & $46 \%$ & $18 \%$ & $36 \%$ \\
\hline
\end{tabular}

Fig. 9, in which the colours of the lines have the same meaning as in panel a. In contrast to the profiles in panel a, $\mathrm{N}_{\mathrm{m}} \mathrm{E}$ in panel b shows no remarkable variations, whilst the density drop in the $\mathrm{F}$ layer is clear. This exemplifies the fact that $\mathrm{N}_{\mathrm{m}} \mathrm{F}_{2}$ depletion alone can also account for presence of ELDI, and such events are regarded as type 2. Panel c shows the third type of ELDI, during the formation of which an enhancement of $35 \%$ in $\mathrm{N}_{\mathrm{m}} \mathrm{E}$ was accompanied by a reduction of $25 \%$ in $\mathrm{N}_{\mathrm{m}} \mathrm{F}_{2}$. The most likely explanation for such an event thus seems to be the combination of (1) and (2).

Density profiles during and around the ELDI events observed by the two radars during 2009-2011 were carefully checked one by one in order to divide these events into the aforementioned three types. The criterion used for identifying the first two types of ELDIs are extremely conservative, limiting the variation of density peak to reductions and enhancements of over $20 \%$, with no overlap between error bars. The distribution of the various types of ELDIs is listed in Table 1 for the EISCAT and ESR radars, respectively, with entries corresponding to their percentages of ELDI events which correspond to each type. It can be seen that ELDIs of types 1 and 3 are more frequently observed than type 2 at both sites. This is easy to understand for the EISCAT mainland radar, since ELDIs appear frequently around magnetic local midnight in conditions dominated by the auroral oval (Fig. 7), during which period the ionospheric E layer is likely to be significantly enhanced by auroral precipitation. For the ESR radar, however, ELDIs are more often observed around magnetic local noon, during which period the cusp is characterized by soft electron precipitation. Soft auroral precipitation in the cusp region tends to cause extra ionization in 

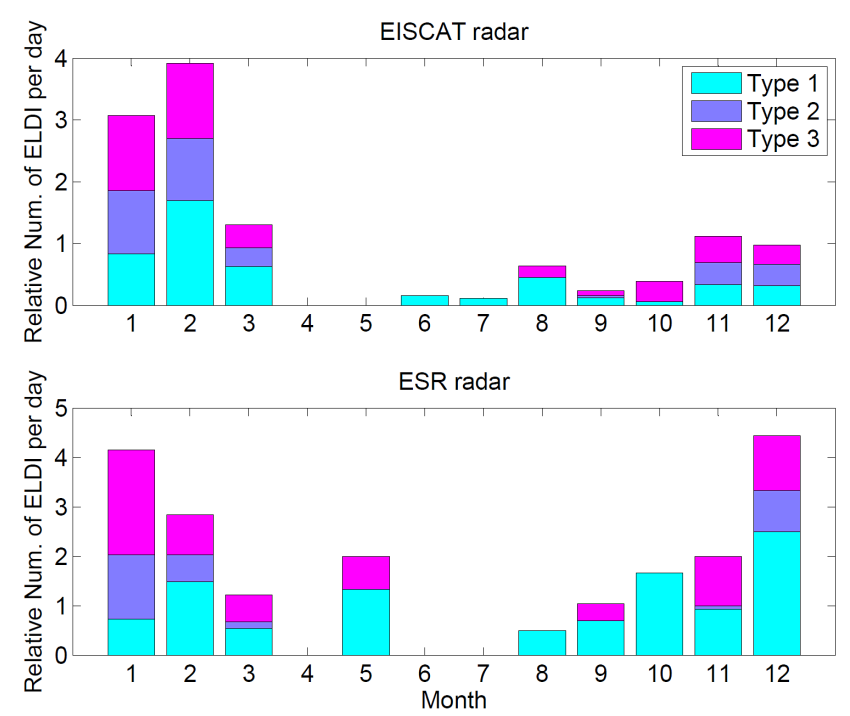

Figure 10. Number of ELDIs of various types in each month at EISCAT/ESR sites during 2009-2011.

ionospheric $\mathrm{F}$ region. This is probably the reason why ELDIs of type 2 are least frequently detected at ESR site.

The relative numbers of the three types of ELDIs for each month and hour are displayed in Figs. 10 and 11, respectively. These distributions were obtained by normalizing the numbers of the various types of ELDIs to the number of observations in Fig. $1 \mathrm{~b}$ and $\mathrm{c}$ for both radars, respectively. From Fig. 10, ELDIs of type 1 and 2 exhibit quite similar seasonal variations at the EISCAT mainland site, with type 2 being somewhat more likely to be detected in December and January at the ESR latitude.

As far as diurnal variation is concerned (Fig. 11), ELDIs of the three types show slight but detectable differences in their occurrence statistics. In the auroral oval (top panel), ELDIs of type 2 are more often observed before geomagnetic midnight (21:00 UT), whilst the occurrence of type 1 exhibits a peak before and after midnight, respectively. On the nightside, moreover, the occurrence of type 2 ELDI peaks at 19:00 UT (22:00 MLT), being slightly earlier than that of type 1 (which maximizes during periods from 20:00 to 21:00 UT and 23:00 to 01:00 MLT). At ESR latitudes (the bottom panel), however, the diurnal variation of ELDIs is essentially dominated by those of type 1 , while these of type 2 are relatively rarely observed on the nightside (16:0004:00 UT, 19:00-07:00 MLT).

Average values of $\mathrm{N}_{\mathrm{m}} \mathrm{F}_{2}$ and $\mathrm{N}_{\mathrm{m}} \mathrm{E}$, observed by the EISCAT mainland and ESR radars during periods of presence and absence of ELDI are listed in Table 2, as well as their standard deviations. Densities are expressed as multiples of $10^{11} \mathrm{~m}^{-3}$. One can see that an increase of $\sim 150-250 \%$ in $\mathrm{N}_{\mathrm{m}} \mathrm{E}$ was concurrent with a reduction of about $40-60 \%$ in $\mathrm{N}_{\mathrm{m}} \mathrm{F}_{2}$ during the presence of ELDIs. If $\mathrm{N}_{\mathrm{m}} \mathrm{E}$ or $\mathrm{N}_{\mathrm{m}} \mathrm{F}_{2}$ are assumed to be unchanged, ELDIs would not be seen at both
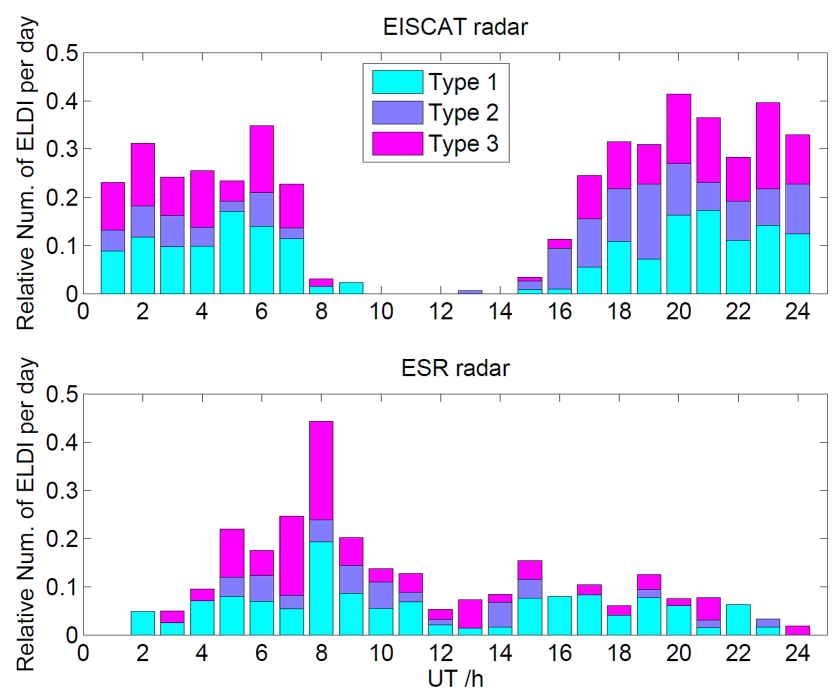

Figure 11. Diurnal variation of various types of ELDIs at EISCAT/ESR sites during 2009-2011 (LT $\approx \mathrm{UT}+1 \mathrm{~h}$, $\mathrm{MLT} \approx \mathrm{UT}+3 \mathrm{~h})$.

Table 2. Average $\mathrm{N}_{\mathrm{m}} \mathrm{F}_{2}$ and $\mathrm{N}_{\mathrm{m}} \mathrm{E}$ during the period of presence and absence of ELDI observed by EISCAT/ESR radars.

\begin{tabular}{lll}
\hline & Presence of ELDI & Absence of ELDI \\
\hline \multirow{2}{*}{ EISCAT site } & $\mathrm{N}_{\mathrm{m}} \mathrm{F}_{2}=1.1 \pm 0.5^{*}$ & $\mathrm{~N}_{\mathrm{m}} \mathrm{F}_{2}=2.5 \pm 0.6$ \\
& $\mathrm{~N}_{\mathrm{m}} \mathrm{E}=1.8 \pm 0.8$ & $\mathrm{~N}_{\mathrm{m}} \mathrm{E}=0.7 \pm 0.7$ \\
\hline \multirow{2}{*}{ ESR site } & $\mathrm{N}_{\mathrm{m}} \mathrm{F}_{2}=0.9 \pm 0.5$ & $\mathrm{~N}_{\mathrm{m}} \mathrm{F}_{2}=1.5 \pm 0.8$ \\
& $\mathrm{~N}_{\mathrm{m}} \mathrm{E}=1.4 \pm 0.9$ & $\mathrm{~N}_{\mathrm{m}} \mathrm{E}=0.4 \pm 0.6$ \\
\hline
\end{tabular}

* with unit of $10^{11} \mathrm{~m}^{-3}$

sites. For example, for a value of $\mathrm{N}_{\mathrm{m}} \mathrm{F}_{2}=2.5 \times 10^{11} \mathrm{~m}^{-3}$ at EISCAT, the requirement for $\mathrm{N}_{\mathrm{m}} \mathrm{E}>\mathrm{N}_{\mathrm{m}} \mathrm{F}_{2}$ cannot be met, even if $\mathrm{N}_{\mathrm{m}} \mathrm{E}$ increases from $0.7 \times 10^{11} \mathrm{~m}^{-3}$ up to $1.8 \times$ $10^{11} \mathrm{~m}^{-3}$. If $\mathrm{N}_{\mathrm{m}} \mathrm{E}$ is assumed to be $0.7 \times 10^{11} \mathrm{~m}^{-3}$, conversely, an $\mathrm{N}_{\mathrm{m}} \mathrm{F}_{2}$ depletion down to $1.1 \times 10^{11} \mathrm{~m}^{-3}$ cannot meet the requirement either. On average, therefore, neither (1) nor (2) alone could lead to the presence of these observed ELDIs. This could also be inferred from Table 1, in which ELDIs of type 1 are less than $50 \%$ and type 2 are no higher than $30 \%$ of the observed events. This suggests that an increase of $\mathrm{N}_{\mathrm{m}} \mathrm{E}$ and a reduction of $\mathrm{N}_{\mathrm{m}} \mathrm{F}_{2}$ can both play a critical role in the formation of ELDI.

\section{Summary}

A survey of the characteristics of ELDI events in the auroral oval and the cusp/polar cap during solar minimum has been carried out, using field-aligned observations from groundbased IS radars. ELDI events have been identified, based on a requirement that their durations should be no shorter than 6 min. 
The occurrence of ELDIs exhibits clear seasonal variations in the auroral oval, with peaks in the winter and early spring. ELDIs are observed most often around geomagnetic local midnight at auroral latitudes, and around local noon at the ESR site. Our results also reveal that ELDI appears to be a sporadic rather than a regular phenomenon in the dark polar region.

The duration of ELDI events exhibits remarkable spatial variations, being about $30 \mathrm{~min}$ at Troms $\varnothing$ on average, but only about a half of this at Svalbard. The thickness of the $\mathrm{E}$ layer, however, as well as $\mathrm{H}_{\mathrm{m}} \mathrm{E}$ and the ratio of $\mathrm{N}_{\mathrm{m}} \mathrm{E} / \mathrm{N}_{\mathrm{m}} \mathrm{F}_{2}$ exhibit similar distribution at both sites. Curve fitting suggests that the means of these parameters at the two sites are quite close during the presence of ELDIs, showing no significant spatial variations.

Case-by-case analysis confirms that either ionization enhancements in the $\mathrm{E}$ layer or density depletions in the $\mathrm{F}$ layer alone can lead to the presence of ELDI, whilst events of type 1 are more frequently observed than type 2 at both sites. The occurrences of ELDI events of the various types exhibit slight but detectable difference between the two sites. We suggest that both $\mathrm{E}$ region enhancement and $\mathrm{F}$ region density depletion play a critical role in ELDI formation in polar region.

Acknowledgements. We are grateful to the director and staff of the EISCAT Scientific Association for providing the radar facilities and assistance with making the observations. EISCAT is an international scientific association funded by the research councils of China, Finland, Germany, Japan, Norway, Sweden and the UK. This work was jointly supported by Natural Sciences Foundation of China (No. 41374159; 41221003; 41431073) and Specialized Research Fund for Key Laboratory of Polar Science of the State Oceanic Administration (No. KP201002).

Topical Editor S. Milan thanks A. Kavanagh and one anonymous referee for their help in evaluating this paper.

\section{References}

Burch, J. L., Lennartsson, W., Hanson, W. B., Heelis, R. A., Hoffman, J. H., and Hoffman, R. A.: Properties of spikelike shear flow reversals observed in the auroral plasma by Atmosphere Explorer C, J. Geophys. Res., 81, 3886-3896, 1976.
Cai, H. T. and Ma, S. Y.: Initial study of inversion method for estimating energy spectra of auroral precipitating particle from ground-based IS radar observations, Chinese J. Geophys.-Ch., 50, 10-17, 2007 (in Chinese).

Cai, H. T., Ma, S. Y., Fan, Y., Liu, Y. C., and Schlegel, K.: Climatological features of electron density in the polar ionosphere from long-term observations of EISCAT/ESR radar, Ann. Geophys., 25, 2561-2569, doi:10.5194/angeo-25-2561-2007, 2007.

Farley, D. T., Balsey, B. B., Woodman, R. F., and McClure, J. P.: Equatorial spread F: Implications of VHF radar observations, J. Geophys. Res., 75, 7199-7216, 1970.

Hultqvist, B., Øieroset, M., Paschmann, G., and Treumann, R.: Processes leading to plasma losses into the high-latitude atmosphere, in: Magnetospheric Plasma Sources and Losses, edited by: Hultqvist, B., Øieroset, M., Paschmann, G., and Treumann, R., Space Sciences Series of ISSI, Springer Netherlands, 1999.

Liang, P. H.: F2 ionization and geomagnetic latitudes, Nature, 160, 642-643, 1947.

Mathews, J. D.: Sporadic E: current views and recent progress, J. Atmos. Sol.-Terr. Phy., 60, 413-435, 1998.

Mayer, C. and Jakowski, N.: Enhanced E-layer ionization in the auroral zones observed by radio occultation measurements onboard CHAMP and Formosat-3/COSMIC, Ann. Geophys., 27, 12071212, doi:10.5194/angeo-27-1207-2009, 2009.

Rees, M. H.: Auroral Ionization and Excitation by Incident Energetic Electrons, Planet Space Sci., 11, 1209-1218, 1963.

Strickland, D. J., Meier, R. R., Hecht, J. H., and Christensen, A. B.: Deducing composition and incident electron spectra from ground-based auroral optical measurement, J. Geophys. Res., 94, 13541-13552, 1989.

Wu, Y. W., Liu, R. Y., Zhang, B. C., Wu, Z. S., Xu, S., and Liu, J. M.: Characteris of the E layer dominated ionosphere in the polar regions during polar nights, Chinese Journal of Polar Research, 25, 132-141, 2013.

Yonezawa, T.: The solar-activity and latitudinal characteristics of the seasonal, non-seasonal and semi-annual variations in the peak electron densities of the F2-layer at noon and at midnight in middle and low latitudes, J. Atmos. Terr. Phy., 33, 889-907, 1971.

Yonezawa, T. and Arima, Y.: On the seasonal and non-seasonal annual variations and the semi-annual variation in the noon and midnight electron densityes of the F2-layer in middle latitudes, J. Radio Res. Lab., 6, 293-309, 1959. 